SANDIA REPORT

SAND98-0207 • UC-705

Unlimited Release

Printed January 1998

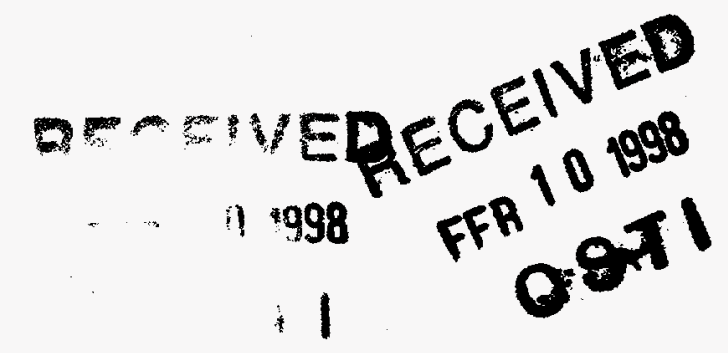

\title{
The Analysis of a Micro-Scale Pump Which Uses Controlled Acoustic Streaming for Fluid Locomotion
}

Jeffrey L. Dohner

Prepared by

Sandia National Laboratories

Albuquerque, New Mexico 87185 and Livermore, California 94550

Sandia is a multiprogram laboratory operated by Sandia Corporation, a Lockheed Martin Company, for the United States Department of

Energy under Contract DE-AC04-94AL85000

Approved for public release; further dissemination unlimited.

सेकk

Find Sandia National Laboratories
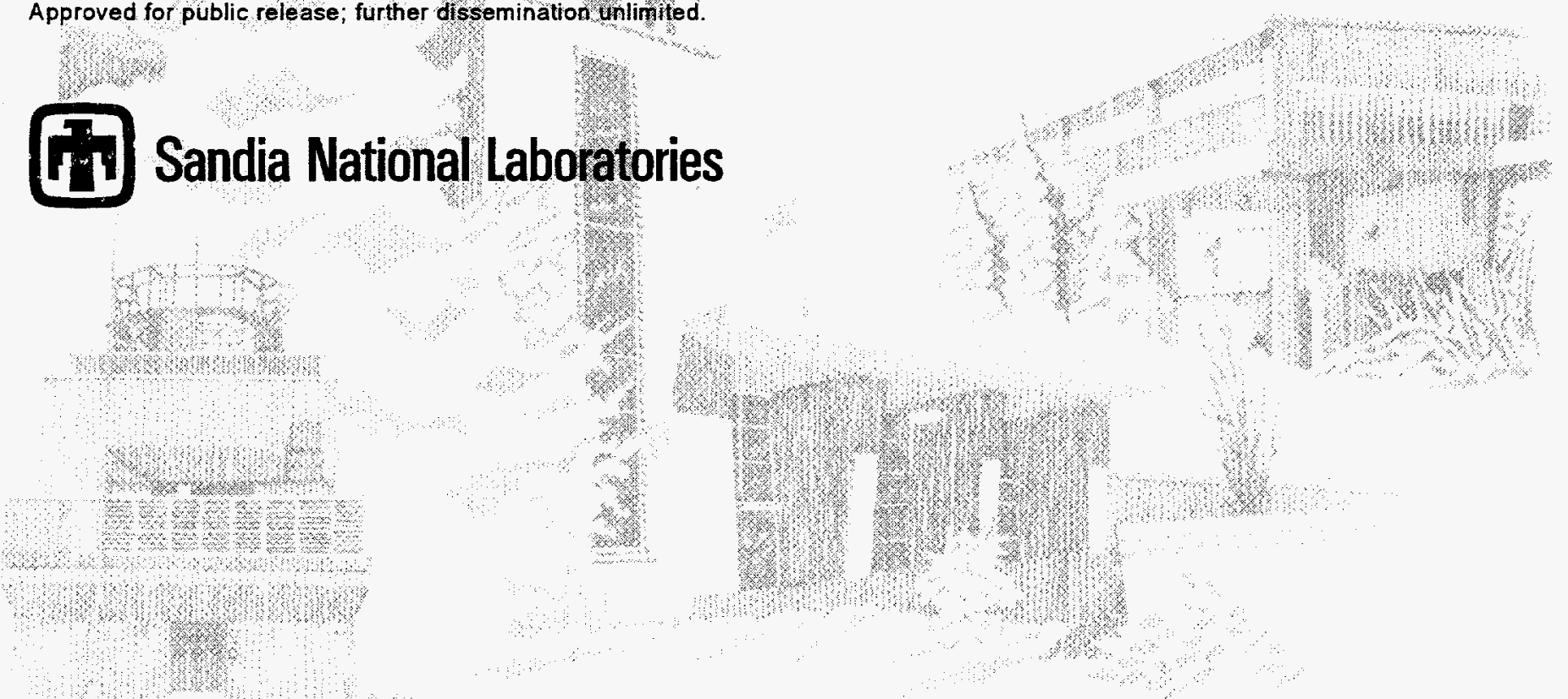
Issued by Sandia National Laboratories, operated for the United States Department of Energy by Sandia Corporation.

NOTICE: This report was prepared as an account of work sponsored by an agency of the United States Government. Neither the United States Government nor any agency thereof, nor any of their employees, nor any of their contractors, subcontractors, or their employees, makes any warranty, express or implied, or assumes any legal liability or responsibility for the accuracy, completeness, or usefulness of any information, apparatus, product, or process disclosed, or represents that its use would not infringe privately owned rights. Reference herein to any specific commercial product, process, or service by trade name, trademark, manufacturer, or otherwise, does not necessarily constitute or imply its endorsement, recommendation, or favoring by the United States Government, any agency thereof, or any of their contractors or subcontractors. The views and opinions expressed herein do not necessarily state or reflect those of the United States Government, any agency thereof, or any of their contractors.

Printed in the United States of America. This report has been reproduced directly from the best available copy.

Available to DOE and DOE contractors from

Office of Scientific and Technical Information

P.O. Box 62

Oak Ridge, TN 37831

Prices available from (615) 576-8401, FTS 626-8401

Available to the public from

National Technical Information Service

U.S. Department of Commerce

5285 Port Royal Rd

Springfield, VA 22161

NTIS price codes

Printed copy: A03

Microfiche copy: A01

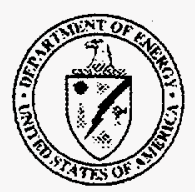




\title{
The Analysis of a Micro-Scale Pump which uses Controlled Acoustic Streaming for Fluid Locomotion
}

\author{
Jeffrey L. Dohner \\ Structural Dynamics Department \\ Sandia National Laboratories \\ P.O.Box 5800 \\ Albuquerque, NM 87185-0439
}

\begin{abstract}
In this report the analysis of a micro-scale pump is described. This micro-pump uses active control to produce a distributed body force in a fluid micro-channel. The desired effect of this body force is to drive fluid through the channel. Limitations, assumptions, and design parameters are discussed.

The mathematical analysis of pump dynamics is explained in detail. A perturbation analysis is used on the equations of mass, momentum and state to produce equations of motion for first and second order effects. The first order effects are described by linear wave motion in the fluid and are found by using integral equation methods. The second order effects are driven by body forces resulting from first order effects. Thus, by controlling the production of wave motion in the channel, second order excitation can also be controlled.

This report is all theory and therefore needs experimental validation. Although many of the assumptions used in this report have been used elsewhere in the literature and have been found to be sufficient, there are many aspects of the problem which have been left unresolved. In particular, flow separation in the fluid channel is a critical problem. If the fluid does not separate, pumping will occur through the channel, however, if internal or external forces are not sufficient to stop separation, this type of pump will not function.
\end{abstract}

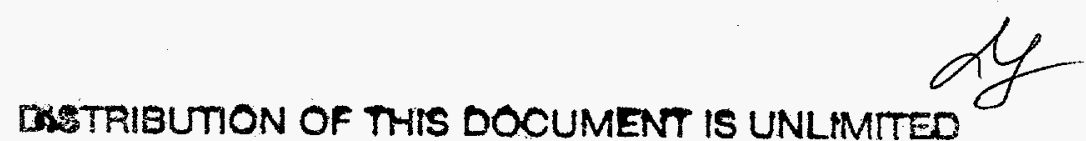


Intentionally Left Blank 


\section{DISCLAIMER}

Portions of this document may be illegible electronic image products. Images are produced from the best available original document. 


\section{Acknowledgment}

I would like to thank Dr. Stephen Martin, Dr. Mike Butler, and Dr. Gregory Frye for their astute insight into this difficult subject. I would also like to thank Dr. Terry Michalske for the additional funding required to complete this work, and Dr. Daniel Segalman for his superlative technical expertise. 
Intentionally Left Blank 


\section{Contents}

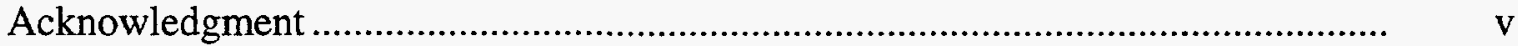

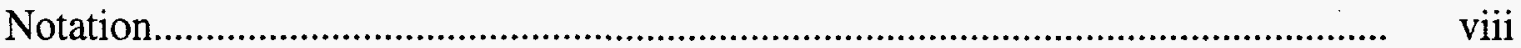

\section{The Analysis of a Micro-Scale Pump which uses Controlled Acoustic Streaming for Fluid Locomotion}

1. Introduction.

2 Pump Geometry

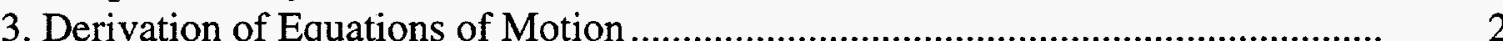

4. Solution of the Equations of Motion................................................................. 5

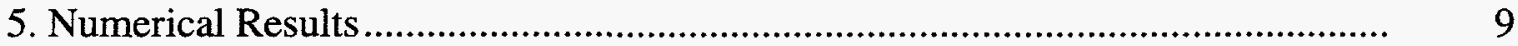

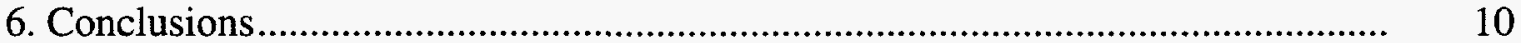

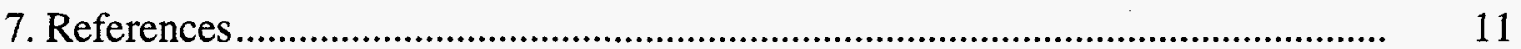

\section{Figures}

Figure 1: Acoustic pump geometry......

Figure 2. Boundary element discretization of Figure 1 section A-A.......................... 5

Figure 3: Pressure difference across the pump for blocked flow and variable amplitude and phase of the left source...

Figure 4: $\mathrm{x}$-direction velocity along the center line of the channel................................ 10

Figure 5: Body force in the $\mathrm{x}$ direction as a function of distance along the center line.... 11 Figure 6: Pressure drop along the length of channel versus locations of the sources.... 11

\section{Tables}

Table 1: Geometric properties. 9

Table 2: Fluid properties. 


\section{Notation}

$l$ - channel length

$h$ - channel height

$w$ - source width

$l_{w}$ - source location from center line

$\rho$ - density of channel fluid

$D$ - velocity of fluid particle

$P$ - fluid pressure

$\mu$ - shear coefficient of viscosity

$\mu_{v}$ - expansion coefficient of viscosity

$s$ - specific entropy

$\rho_{0}, \rho_{1}, \rho_{2}$ - perturbations of density

$P_{0}, P_{1}, P_{2}$ - perturbations of pressure

$\vec{v}_{0}, \vec{v}_{1}, \vec{v}_{2}$ - perturbations of velocity

$c_{0}=\sqrt{\mathrm{B} / \rho_{0}}-$ ambient sound speed

$B$ - bulk modulus of fluid

$F$ - body force excitation

$\gamma=\mu / \rho_{0}$

$\gamma^{\prime}=\frac{1}{\rho_{0}}\left(\mu+\frac{3}{4} \mu_{v}\right)$

$\phi$ - dilatational potential

$\vec{\psi}$ - shear potential

$\vec{v}_{1}=\overrightarrow{V_{1}} e^{j \omega t}$

$\phi=\Phi e^{j \omega t}$

$\vec{\psi}=\dot{\Psi} e^{j \omega t}$ $k_{l}$ - dilatational wave number

$k_{s}$ - shear wave number

$\omega$ - circular frequency

$c_{l}$-dilatational sound speed

$c_{s}$ - shear sound speed

$\langle f\rangle$ - time averaged response of $f$

$C$ - solid angle coefficient

$G\left(\grave{r}_{0} \mid(\grave{r}, k)\right)$ - Green's function

$\Omega$ - fluid domain

$\Gamma$ - surface of fluid domain

$F_{0}$ - vector pointing to a point in $\Omega$

$\vec{r}$ - vector pointing to a point in $\Gamma$

$\vec{R}=\vec{r}_{0}-\vec{r}$

$C$ - solid angle on surface

$H_{0}(x)-0^{t h}$ order Hankel function

$\Psi_{z}$ - shear potential for 2-D problems

$\tilde{\Phi}, \tilde{\Psi}, \frac{\partial \dot{\Phi}}{\partial n}, \frac{\partial \tilde{\Psi}}{\partial n}$ - solution vectors

$\tilde{G}_{\Phi}, \tilde{H}_{\Phi}, \tilde{G}_{\Psi}, \tilde{H}_{\Psi}$ - influence matrices

$\tilde{G}_{\Phi_{o b s}}, \tilde{H}_{\Phi_{o b s}}, \tilde{G}_{\Psi_{o b s}}, \tilde{H}_{\Psi_{o b s}}$ - influence matrices

$\tilde{\Phi}_{o b s}, \tilde{\Psi}_{o b s}$ - solution vectors

$\Delta_{i}$ - length of $i^{\text {th }}$ element

$n_{p}$ - number of elements

$\overrightarrow{\Delta x}, \overrightarrow{\Delta y}$ - perturbation in $\mathrm{x}$ and $\mathrm{y}$ 


\section{INTRODUCTION}

In this paper the analysis of an acoustic micro-pump is presented. Unlike previous micropumps which are comprised of oscillating diaphragms and a series of complex values, this pump is unique in that it is based on the concepts of active control and acoustic streaming.

Micro-scale pumps have many applications. They can be used to drive micro-cooling systems which can be used to eliminate detrimental heat in circuitry and can be used to power micro-fluid systems which can be used as an alternative to micro-electronics. They can also be integrated into motion control systems for the purpose of moving objects in micro-space.

Recently, a few authors [1,2] have suggested using the phenomena of acoustic streaming to build micro-pumps. Pumps operating by streaming would have few moving parts and could be easily constructed. Acoustic streaming results from Reynolds stress in a fluid. Reynolds stress can be produced by an acoustic wave with spatially varying amplitude. By controlling the acoustic waves which produce Reynolds stress, fluid motion can also be controlled.

Acoustic streaming is a well known phenomena. Nyborg [3] used a perturbation method and time averaging to explain how a damped acoustic plane wave can produce streaming. Lighthill [4] expanded upon Nyborg's explanation by quantifying the significance of various terms in the streaming equations. In this paper not all of the terms discussed by Lighthill will be used. Only those terms that are necessary to show how active control can be used to move fluid through a channel will be retained.

R.M. Moroney, R.M. White, and R.T. Howe [1] constructed a pumping device driven by a flexural plate wave source. This device produced streaming by using the natural attenuation of evanescent shear waves to create a Reynolds stress. S. Shiokawa and Y. Matsui [2] showed that a leaky surface wave could produce a larger Reynolds stress in the fluid than a surface wave without leakage.

In R.M. Moroney, R.M. White and R.T.Howes' work, Reynold's stress was the result of wave distortion due to natural attenuation. In S. Shiokawa and Y. Matsuis' work this distortion was enhanced by attenuation due to leakage. In this work distortion will result from wave interference produced by using active control.

\section{PUMP GEOMETRY}

The acoustic pump under consideration is shown in Figure 1. This pump consist of two substrates with an open channel cut between them. A cross-sectional view of this channel is shown in section A-A. The channel is of length $l$ and of height $h$ and it is assumed that the fluid in the channel is Newtonian. On either side of the channel is a set of velocity sources. Sources are of width $w$ and are $l_{w}$ from the center of the channel. Opposing sources are driven 180 degrees out of phase. In this paper opposing pairs will be treated as a single source. The pair of sources to the left of the center of the channel will be referred to as the left source, and the pair of sources to the right of the center of the channel will be 
referred to as the right source. As will be shown, by varying the relative phasing between right and left sources, a net time averaged pressure gradient can be produced across the channel.

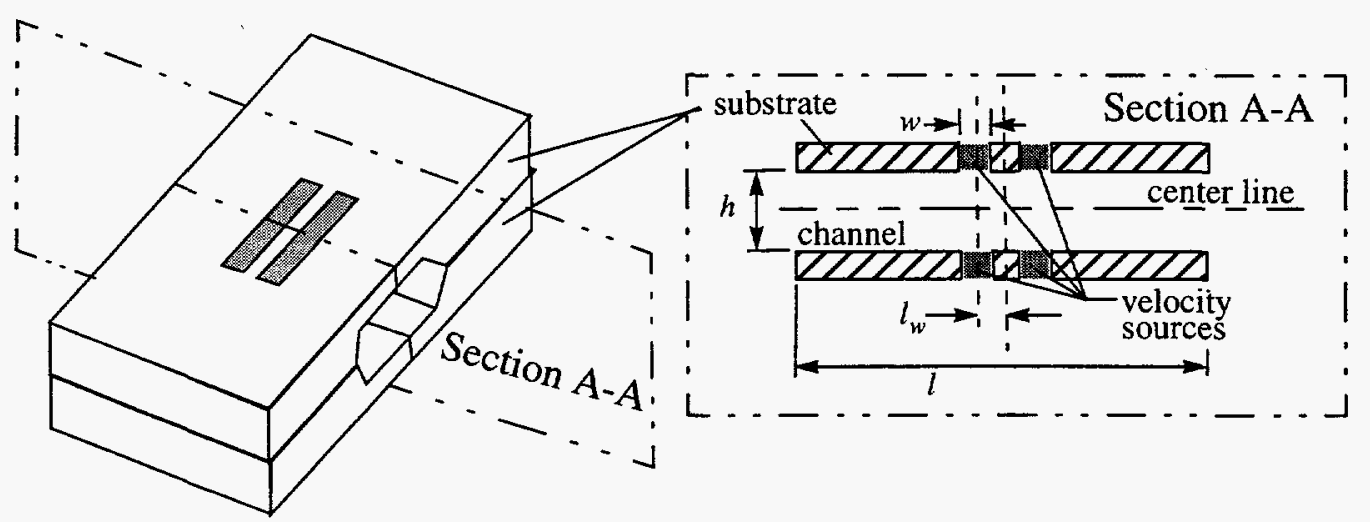

Figure 1: Acoustic pump geometry

\section{DERIVATION OF EQUATIONS OF MOTION}

In the following section first and second order equations of motion for a Newtonian fluid are derived. Much of this information has been accumulated from Temkin [5], Nyborg [3] and Lighthill [4].

The equations of mass, momentum and state for a Newtonian fluid are given by

$$
\begin{aligned}
& \text { (mass) } \frac{\partial \rho}{\partial t}+\nabla \cdot(\rho \grave{\nu})=0
\end{aligned}
$$

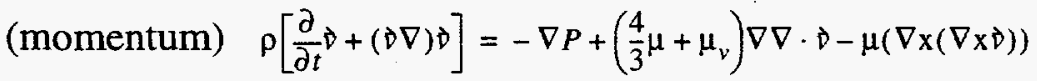

$$
\begin{aligned}
& \text { (state) } \quad P=P(\rho, s)
\end{aligned}
$$

where $\rho$ is the density of the fluid, $\vec{\nu}$ is the velocity of a fluid particle, $\mu$ is the shear coefficient of viscosity, $\mu_{v}$ is the expansion coefficient of viscosity, and $s$ is the specific entropy.

Substituting the perturbation expansion

$$
\begin{aligned}
& \rho=\rho_{0}+\rho_{1} \varepsilon+\rho_{2} \varepsilon^{2} \ldots \\
& P=P_{0}+P_{1} \varepsilon+P_{2} \varepsilon^{2} \ldots \\
& \grave{\nu}=\nu_{0}+\nu_{1} \varepsilon+\nu_{2} \varepsilon^{2} \ldots
\end{aligned}
$$

into (1), (2), and (3) and assuming that $\vec{\nu}_{0}=0$ (i.e., no externally driven flow) gives to $\varepsilon$ order 


$$
\begin{gathered}
\text { (mass) } \frac{\partial \rho_{1}}{\partial t}+\rho_{0} \nabla \cdot \vec{v}_{1}=0 \\
\text { (momentum) } \rho_{0} \frac{\partial}{\partial t} \overrightarrow{v_{1}}=-\nabla P_{1}+\left(\frac{4}{3} \mu+\mu_{v}\right) \nabla \nabla \cdot \overrightarrow{v_{1}}-\mu \nabla \times \nabla \times \overrightarrow{v_{1}} \\
\text { (state) } P_{1}=c_{0}^{2} \rho_{1}
\end{gathered}
$$

where $c_{0}=\left(\frac{\partial P}{\partial \rho}\right)_{s}=\sqrt{\frac{\mathrm{B}}{\rho_{0}}}$ and $\mathrm{B}$ is the bulk modulus of the fluid.

To $\varepsilon^{2}$ order this perturbation expansion gives (momentum)

$$
\rho_{0} \frac{\partial}{\partial t} \vec{v}_{2}=-\nabla P_{2}+\left(\frac{4}{3} \mu+\mu_{v}\right) \nabla \nabla \cdot \vec{v}_{2}-\mu \nabla \times \nabla \times v_{2}-\frac{\partial}{\partial t} \rho_{1} \vec{v}_{1}-\rho_{0}\left(\vec{v}_{1} \cdot \nabla\right) \vec{v}_{1}-\rho_{0} \vec{v}_{1}\left(\nabla \cdot \vec{v}_{1}\right)
$$

Taking the time averaged of (10) and noticing that, as stated by Lighthill [4], $\left\langle\frac{\partial}{\partial t} \rho_{1} u_{1}\right\rangle-0$, equation (10) becomes

$$
\left\langle-\nabla P_{2}+\left(\frac{4}{3} \mu+\mu_{v}\right) \nabla \nabla \cdot \overrightarrow{v_{2}}-\mu \nabla \times \nabla \times \overrightarrow{v_{2}}\right\rangle=-\vec{F}
$$

where $\langle f\rangle$ is the time average of $f$ and

$$
\vec{F}=\rho_{0}\left\langle\left(\vec{\nu}_{1} \cdot \nabla\right) \vec{\nu}_{1}+\vec{\nu}_{1}\left(\nabla \cdot \vec{\nu}_{1}\right)\right\rangle .
$$

The function $\left(\vec{\nu}_{1} \cdot \nabla\right) \vec{\nu}_{1}+\vec{\nu}_{1}\left(\nabla \cdot \vec{\nu}_{1}\right)$ is the Reynold's stress vector.

Assuming that $\left\langle\nabla \cdot \dot{v}_{2}\right\rangle=0$ (for second order channel flow)

$$
\mu \nabla^{2}\left\langle\vec{v}_{2}\right\rangle=-\vec{F}+\nabla\left\langle P_{2}\right\rangle .
$$

Equation (13) is Laplace's equation driven by a right hand side comprised of first order terms and the gradient of the time averaged second order pressure. The vector function $\vec{F}$ represents a body force driving the second order equation of motion. To calculate this body force, the first order solution (7), (8), and (9) must be solved for and substituted into (12). This first order solution can be represented in terms of linear dilatational and shear waves. By controlling these waves in the fluid, the body force, $\vec{F}$, can be controlled and therefore, so can the second order time averaged motion of fluid through the channel.

This first order solution can be represented in terms of linear dilatational and shear waves. Combining equation (7), (8) and (9) gives

$$
\frac{\partial^{2} \grave{\triangleright}_{1}}{\partial t^{2}}-c_{0}^{2}\left(1+\frac{4}{3} \frac{\gamma^{\prime}}{c_{0}^{2}} \frac{\partial}{\partial t}\right) \nabla\left(\nabla \cdot \vec{\triangleright}_{1}\right)=-\gamma \nabla x \nabla x \grave{1}_{1}
$$


where $\gamma^{\prime}=\frac{1}{\rho_{0}}\left(\mu+\frac{3}{4} \mu_{v}\right)$ and $\gamma=\frac{\mu}{\rho_{0}}$. The velocity $\nu_{1}$ can be written as

$$
\vec{v}_{1}=\nabla \phi+\nabla x \vec{\varphi}
$$

where $\nabla \mathrm{X} \phi$ and $\nabla \cdot \vec{\psi}$. Substituting (15) into (14) and taking the gradient of the result gives

$$
\frac{\partial^{2} \phi}{\partial t^{2}}-c_{0}^{2}\left(1+\frac{4}{3} \frac{v^{\prime}}{c_{0}^{2}} \frac{\partial}{\partial t}\right) \nabla^{2} \phi=0 .
$$

Substituting (15) into (14) and taking the curl of the result gives

$$
\frac{\partial^{2}}{\partial t^{2}} \vec{\psi}-v \nabla^{2} \vec{\psi}=0
$$

Equation (16) is the wave equation for dilatational motion in the fluid, and (17) is the wave equation for shear motion in the fluid.

The temporal transform of (15), (16) and (17) results in

$$
\begin{gathered}
\vec{\nabla}_{1}=\nabla \Phi+\nabla x \Psi \\
\left(\nabla^{2}+k_{l}^{2}\right) \Phi=0,\left(\nabla^{2}+k_{s}^{2}\right) \Psi=0 \\
k_{l}^{2}=\frac{\omega^{2} / c_{o}}{1-j \omega \cdot \frac{4 \gamma^{\prime}}{3 c_{0}}}=\frac{\omega^{2}}{c_{l}^{2}}, k_{s}^{2}=\frac{\omega^{2}}{-j \omega \cdot \gamma}=\frac{\omega^{2}}{c_{s}^{2}},
\end{gathered}
$$

where

$$
\vec{D}_{1}=\operatorname{Re}\left(\overrightarrow{V_{1}} e^{j \omega t}\right), \phi=\operatorname{Re}\left(\Phi e^{j \omega t}\right), \dot{\psi}=\operatorname{Re}\left(\Psi e^{j \omega t}\right)
$$

for harmonic excitation. Equations (19) and (20) can be solved for using a boundary element method. The solution to these equations will produce $\Phi$ and $\dot{\Psi}$. Using this solution and (18), $\nabla$ can also be determined.

Rewriting (12) in terms of $\overrightarrow{V_{1}}$ gives

$$
\vec{F}=\frac{1}{2} \rho_{0}\left\{\operatorname{Re}\left(\vec{\nabla}_{1}\right)\left(\nabla \cdot \operatorname{Re}\left(\vec{\nabla}_{1}\right)\right)+\operatorname{Im}\left(\vec{\nabla}_{1}\right)\left(\nabla \cdot \operatorname{Im}\left(\vec{\nabla}_{1}\right)\right)+\left(\operatorname{Re}\left(\vec{\nabla}_{1}\right) \cdot \nabla\right) \operatorname{Re}\left(\vec{\nabla}_{1}\right)+\left(\operatorname{Im}\left(\vec{\nabla}_{1}\right) \cdot \nabla\right) \operatorname{Im}\left(\vec{\nabla}_{1}\right)\right\}
$$

Thus, with $\vec{\nabla}, \vec{F}$ is determined. For waves propagating in only the $\mathrm{x}$ direction, (26) becomes

$$
F_{x}=\rho_{0}\left\{\operatorname{Re}\left(V_{x}\right) \operatorname{Re}\left(\frac{\partial V_{x}}{\partial x}\right)+\operatorname{Im}\left(V_{x}\right) \operatorname{Im}\left(\frac{\partial V_{x}}{\partial x}\right)\right\}
$$

where $\overrightarrow{V_{1}}=V_{x} \hat{i}, \vec{F}=F_{x} \hat{i}$ is a vector in the $\mathrm{x}$ direction. 


\section{SOLUTION OF THE EQUATIONS OF MOTION}

Equations (19), (20) and (13) are the equations of motion for the fluid. Equation (19) and (20) can be used to solve for $\Phi$ and $\Psi$ using a boundary element method. With $\Phi$ and $\dot{\Psi}$, (18) can be used to solve for $\vec{\nabla}_{1}$, and with $\vec{\nabla}_{1}$, (26) can be used to solve for $\vec{F}$. With $\vec{F}$, equation (13) can be used to solve for the time average flow of fluid in the channel, $\left\langle\vec{\nu}_{2}\right\rangle$, or the net time averaged pressure gain across the channel $\nabla\left\langle P_{2}\right\rangle$. In this section a solution for $\nabla\left\langle P_{2}\right\rangle$ is outlined.

Assuming that the width of the channel is large compared to its thickness, a two dimensional plane stress analysis is appropriate. The surface of the Figure 1 fluid domain can be discretized as shown in Figure 2. In two dimensional space, the integral representations of (19) and (20) are given by

$$
\begin{aligned}
& C \phi\left(\grave{P}_{0}\right)=-\int_{\Gamma}\left\{G\left(\grave{r}_{0} \mid \vec{r}, k_{l}\right) \frac{\partial}{\partial n} \Phi(\vec{r})-\frac{\partial}{\partial n} G\left(\grave{r}_{0} \mid \vec{F}, k_{l}\right) \Phi(\vec{r})\right\} d \Gamma \\
& C \Psi_{z}\left(\vec{r}_{0}\right)=-\int_{\Gamma}\left\{G\left(\vec{r}_{0} \mid \vec{p}, k_{s}\right) \frac{\partial}{\partial n} \Psi_{z}(\vec{p})-\frac{\partial}{\partial n} G\left(\vec{r}_{0} \mid \vec{p}, k_{s}\right) \Psi_{z}(\vec{p})\right\} d \Gamma
\end{aligned}
$$

where $\Gamma$ is the surface of the fluid domain, $\Omega, G\left(\vec{p}_{0} \mid \vec{p}, k\right)=\frac{1}{\sqrt{2 \pi}} H_{0}(k R), R=|\vec{R}|, \vec{k}=\vec{p}_{0}-\vec{p}$, $C=1$ if $\xi_{0} \notin \Gamma, C=1 / 2$ if $\xi_{0} \in \Gamma, H_{0}$ is a zeroth order Hankel function and $\Psi=\Psi_{2} \hat{k}$ where $\hat{k}$ is a unit vector normal to the analysis plane.

As shown in Figure 2, vector notation can be used to define points in the channel and on the channel surface. The surface $\Gamma$ can be represented as the union of a number of subsurfaces where

$$
\Gamma=\Gamma_{\text {top }_{\text {rigid }}} \cup \Gamma_{\text {top }_{s 1}} \cup \Gamma_{\text {top }_{s 2}} \cup \Gamma_{\text {bottom }_{\text {rigid }}} \cup \Gamma_{\text {bottom }_{s 1}} \cup \Gamma_{\text {bottom }_{s 2}} \text {. }
$$

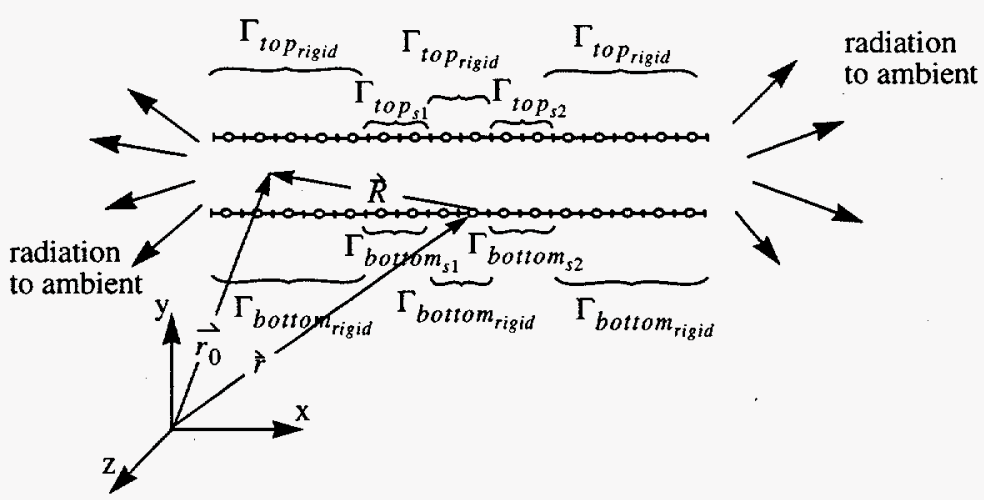

Figure 2: Boundary element discretization of Figure 1 section A-A 
If the surface $\Gamma$ is discretized into a distribution of uniform elements, then using boundary element theory [6] there will be $4 \cdot n_{p}$ unknowns in this problem where $n_{p}$ is the total number of elements. These unknowns are the values of $\Phi\left(\vec{r}_{i}\right), \frac{\partial}{\partial n} \Phi\left(\vec{r}_{i}\right), \Psi_{z}\left(\vec{r}_{i}\right)$ and $\frac{\partial}{\partial n} \Psi_{z}\left(\vec{t}_{i}\right)$ at the center node of each element. Thus, $4 \cdot n_{p}$ unique equations are required to solve for these $4 \cdot n_{p}$ unknowns. These equations come from (28) and (29) and from boundary conditions.

Two $n_{p}$ unique equations can be formed by discretizing (28) and (29) as

$$
\frac{1}{2} \tilde{\Phi}=\tilde{G}_{\Phi} \frac{\tilde{\partial}}{\partial n}-\tilde{H}_{\Phi} \tilde{\Phi}, \frac{1}{2} \tilde{\Psi}=\tilde{G}_{\Psi} \frac{\partial \Psi}{\partial n}-\tilde{H}_{\Psi} \tilde{\Psi}
$$

where

$$
\begin{aligned}
& \tilde{\Phi}=\left[\Phi\left(\grave{r}_{1}\right), \boldsymbol{\Phi}\left(\grave{r}_{2}\right), \ldots\right]^{T}, \tilde{\Psi}=\left[\Psi_{z}\left(\grave{r}_{1}\right), \Psi_{z}\left(\grave{r}_{2}\right), \ldots\right]^{T}, \\
& \frac{\partial \Phi}{\partial n}=\left[\frac{\partial \Phi}{\partial n}\left(\grave{r}_{1}\right), \frac{\partial \Phi}{\partial n}\left(\grave{r}_{2}\right), \ldots\right]^{T}, \frac{\partial \Psi}{\partial n}=\left[\frac{\partial \Psi}{\partial n}\left(\vec{r}_{1}\right), \frac{\partial \Psi}{\partial n}\left(\vec{r}_{2}\right), \ldots\right]^{T},
\end{aligned}
$$

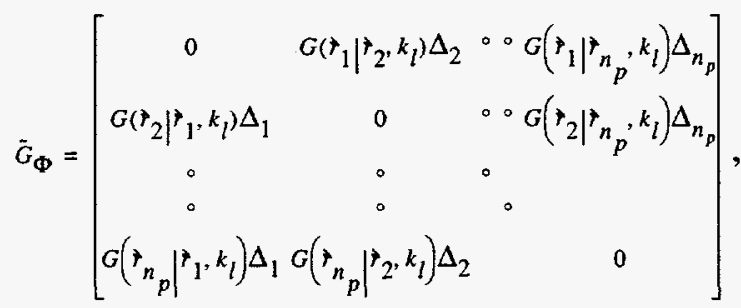

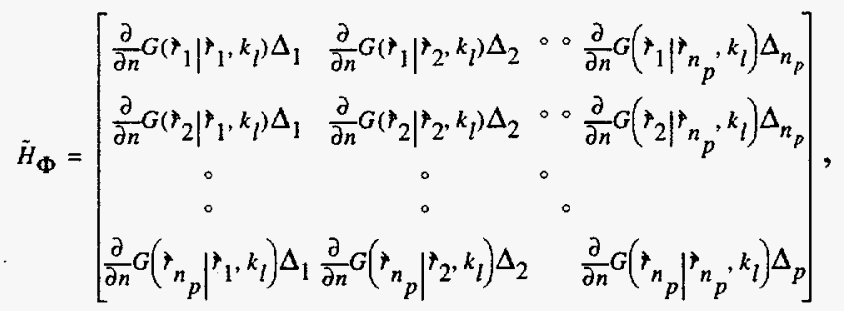

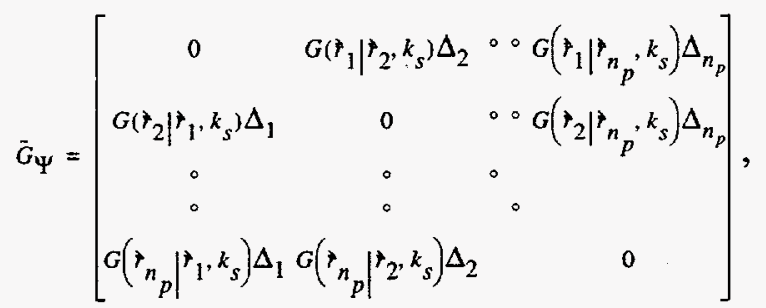

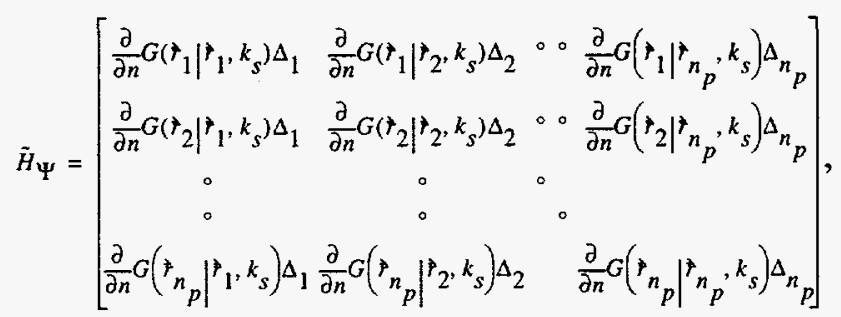

$\Delta_{i}$ is the length of the $i^{\text {th }}$ element and $\left\{\vec{r}_{1}, \vec{r}_{2}, \ldots \vec{t}_{n_{p}}\right\} \in \Gamma$. 
Another $2 \cdot n_{p}$ equations can be formed from the boundary conditions. These boundary conditions are different for each sub-surface of $\Gamma$. On the $\Gamma_{\text {rop } p_{\text {rigid }}}$ and $\Gamma_{\text {bottom }}$ igid $_{\text {ig }}$ surface, the normal velocity is zeros, therefore, from (18)

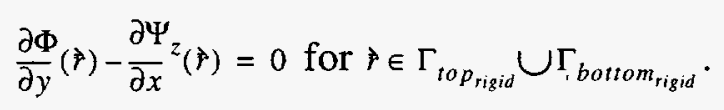

The term $\frac{\partial \Phi}{\partial y}(\gg)$ in (32) is contained in the vector $\frac{\partial \dot{\Phi}}{\partial n}$ and the term $\frac{\partial \Psi}{\partial x}^{k}(\gg)$ in (32) can be determined by using a finite differences.

Velocity sources are also represented as a set of boundary conditions. If $v_{\text {left }}$ is the normal velocity of the left source and $v_{\text {right }}$ is the velocity of source, then

$$
\begin{aligned}
& \frac{\partial \Phi}{\partial y}(\vec{r})-\frac{\partial \Psi}{\partial x}(\vec{r})=-v_{l e f t} \text { for } \vec{r} \in \Gamma_{t o p_{s 1}} \\
& \frac{\partial \Phi}{\partial y}(\grave{r})-\frac{\partial \Psi}{\partial x} z_{(\grave{p})}=v_{\text {left }} \text { for } \grave{\vec{r}} \in \Gamma_{\text {bottom }_{s 1}}
\end{aligned}
$$

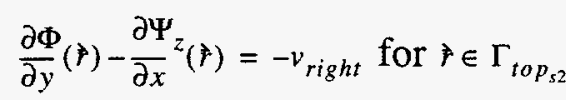

$$
\begin{aligned}
& \frac{\partial \Phi}{\partial y}(\grave{r})-\frac{\partial \Psi}{\partial x}(\grave{r})=v_{r i g h t} \text { for } \vec{p} \in \Gamma_{\text {bottom }_{s z}}
\end{aligned}
$$

Equations (32) to (36) are the boundary conditions for this problem. These conditions along with the $2 n_{p}$ conditions in $(30,31)$ produce enough unique equations to solve for the vectors $\tilde{\Phi}, \tilde{\Psi}, \frac{\partial \dot{\Phi}}{\partial n}$ and $\frac{\partial \dot{\Psi}}{\partial n}$. These vectors contain the potential solutions on the surface of the channel and can be used to calculate potentials at any point in the channel.

From (28) and (29) the potentials at any location in the channel can be calculated by

$$
\begin{gathered}
\tilde{\Phi}_{o b s}=\tilde{G}_{\Phi_{o b b} \partial \tilde{\Phi}}-\tilde{H}_{\Phi_{o b s}} \tilde{\Phi}, \\
\tilde{\Psi}_{o b s}=\tilde{G}_{\Psi_{o b s}} \frac{\partial \tilde{\Psi}}{\partial n}-\tilde{H}_{\Psi_{o b s}} \tilde{\Psi}
\end{gathered}
$$

where

$$
\tilde{\Phi}_{o b s}=\left[\Phi\left(\grave{r}_{1_{o b s}}\right), \Phi\left(\grave{P}_{2_{o b s}}\right), \ldots\right]^{T}, \tilde{\Psi}_{o b s}=\left[\Psi_{z}\left(\vec{P}_{1_{o b s}}\right), \Psi_{z}\left(\grave{P}_{2 o b s}\right), \ldots\right]^{T}
$$

$\left\{\vec{f}_{1_{o b s},} \vec{z}_{2_{o b s}}, \ldots \vec{t}_{n_{o b s}}\right\} \in \Omega, n_{o b s}$ is the number of observation points, 


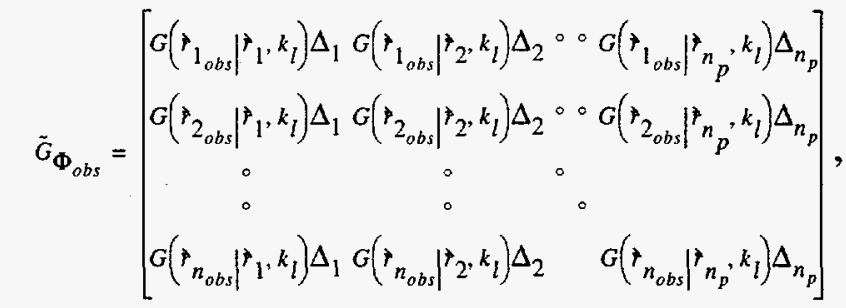

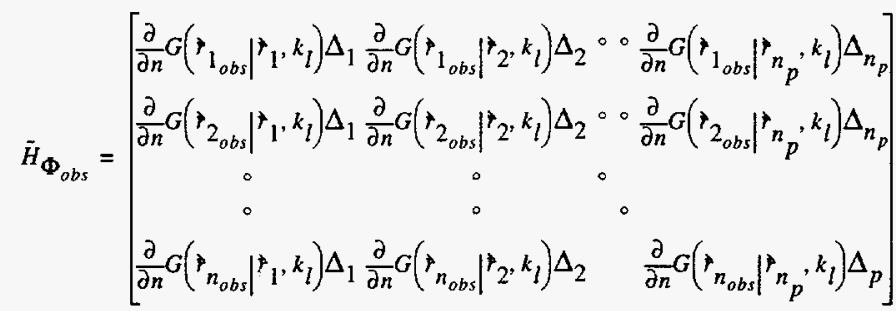

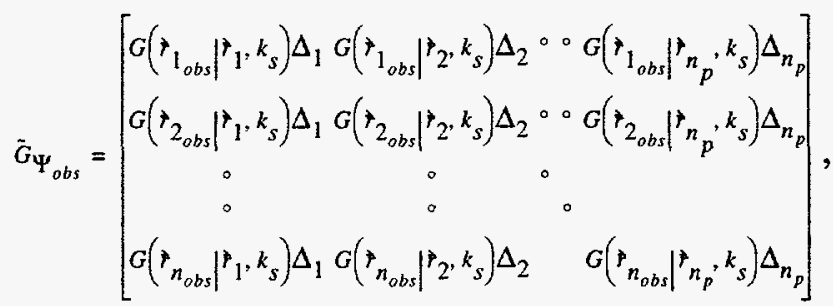

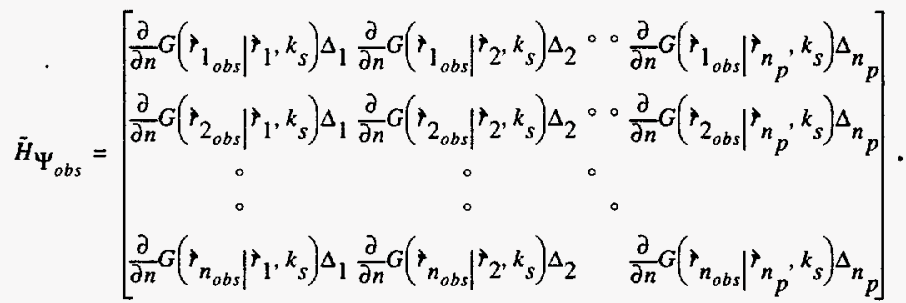

From (37) and (38) the potentials at any point in the channel can be determined. To solve for the body force, $\vec{F}$, the velocity, $\vec{\nabla}_{1}$, must be computed from the spatial derivatives of these potentials. These spatial derivatives can be approximated by using the central difference formulas

$$
\begin{gathered}
\frac{\partial}{\partial x} \Phi\left(\vec{f}_{1_{o b s}}\right)-\frac{\Phi\left(\vec{r}_{1_{o b s}}+\overrightarrow{\Delta x}\right)-\Phi\left(\vec{f}_{1_{o b s}}-\overrightarrow{\Delta x}\right)}{2|\overrightarrow{\Delta x}|} \\
\frac{\partial}{\partial y} \Phi\left(\vec{r}_{1_{o b s}}\right) \sim \frac{\Phi\left(\vec{r}_{1_{o b s}}+\overrightarrow{\Delta y}\right)-\Phi\left(\vec{r}_{1_{o b s}}-\overrightarrow{\Delta y}\right)}{2|\overrightarrow{\Delta y}|} \\
\frac{\partial}{\partial x} \Psi_{z}\left(\vec{r}_{1_{o b s}}\right) \sim \frac{\Psi_{z}\left(\vec{P}_{1_{o b s}}+\overrightarrow{\Delta x}\right)-\Psi_{z}\left(\vec{r}_{1_{o b s}}-\overrightarrow{\Delta x}\right)}{2|\overrightarrow{\Delta x}|} \\
\frac{\partial}{\partial y} \Psi_{z}\left(\vec{l}_{1_{o b s}}\right) \sim \frac{\Psi_{z}\left(\vec{l}_{1_{o b s}}+\overrightarrow{\Delta y}\right)-\Psi_{z}\left(\vec{r}_{1_{o b s}}-\overrightarrow{\Delta y}\right)}{2|\overrightarrow{\Delta y}|}
\end{gathered}
$$

where $\overrightarrow{\Delta x}$ and $\overrightarrow{\Delta y}$ are small amplitude vectors in the $\mathrm{x}$ and $\mathrm{y}$ directions. Using equations (40) to (43) with equation (18), the velocity, $\vec{\nabla}_{1}$, at any point in the domain can be determined. Then using (26), the body force, $\vec{F}$, can be determined. With $\vec{F}$, the time averaged velocity, $\left\langle\vec{v}_{2}\right\rangle$, or the time average pressure gradient, $\nabla\left\langle P_{2}\right\rangle$, can be determined from (13). 


\section{NUMERICAL RESULTS}

Using the geometric and fluid properties given in Table 1 and Table 2 and an excitation frequency of $10^{5} \mathrm{~Hz}$, a first order and second order solution can be determined.

Table 1: Geometric Properties

\begin{tabular}{|c|c|c|c|}
\hline$h$ & $w$ & $l_{w}$ & $l$ \\
\hline \hline $400 \mathrm{um}$ & $1000 \mathrm{um}$ & $1000 \mathrm{um}$ & $18000 \mathrm{um}$ \\
\hline
\end{tabular}

Table 2: Fluid properties (air)

\begin{tabular}{|c|c|c|c|}
\hline$\mu$ & $\mu_{\nu}$ & $\rho_{0}$ & $\mathrm{~B}$ \\
\hline \hline $\begin{array}{l}1.846 \times 10^{-11} \\
\mathrm{~kg} / \mathrm{um} \cdot \mathrm{s}\end{array}$ & $0.65 \cdot \mu$ & $\begin{array}{l}1.77 \times 10^{-7} \\
\mathrm{~kg} \cdot \mathrm{m} / \mathrm{um}^{4}\end{array}$ & $\begin{array}{l}2.135 \times 10^{-7} \\
\mathrm{~N} / \mathrm{um}^{2}\end{array}$ \\
\hline
\end{tabular}

The first order velocity distribution across the channel was found to be almost uniform everywhere except very close to the wall. Thus, it was assumed that acoustic propagation was one dimensional and wall effects contributed little to the second order flow through the channel. Since wave propagation through the pump is one dimensional, the body force, $\vec{F}$, can be approximated by (27).

If the flow of fluid through the pump is blocked but acoustically transparent, a net pressure head, $\Delta P$, develops across the pump. From (13), this pressure is simply the integral of $\vec{F}$ along the length of the pump. This net pressure head is an indication of the ability of the pump to force fluid through the channel assuming that the fluid in the pump does not separate.

Letting $v_{r i g h t}=1.0 u m / s$ then $v_{\text {left }}$ can be varied to determine $\Delta P$ as a function of amplitude and phase. Figure 3 shows $v_{\text {left }}$ for various amplitude between 0 and 1.0, and phases between 0 and $2 \pi$ radians. Notice that maximum $\Delta P$ occurs when $v_{l e f t}=1.0 e^{j \frac{\pi}{2}} u m / s$ and when $v_{\text {left }}=1.0 e^{j \frac{3 \pi}{2}} u m / s$ where the first maximum corresponds to pumping to the left, and the second maximum corresponds to pumping to the right

In Figure 4 the $x$-direction first order acoustic velocity of the fluid at the center of the channel can be seen for $v_{\text {left }}=1.0 e^{j \frac{3 \pi}{2}} u m / s$. Notice that the waves from the two sources cancel to produce waves propagating from the sources in opposite directions with different amplitudes. Thus, the amplitude of wave velocity in the channel changes substantially near the sources. 


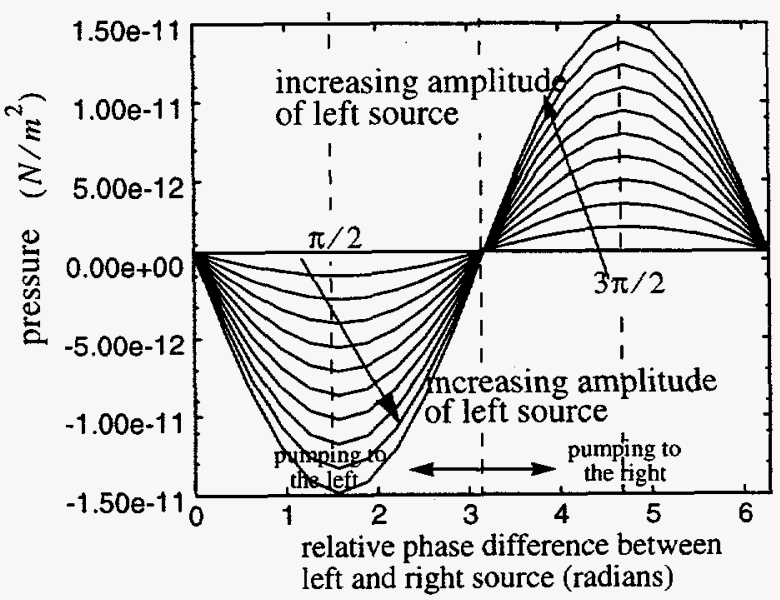

Figure 3: Pressure difference across pump for block flow and variable amplitude and phase of left source

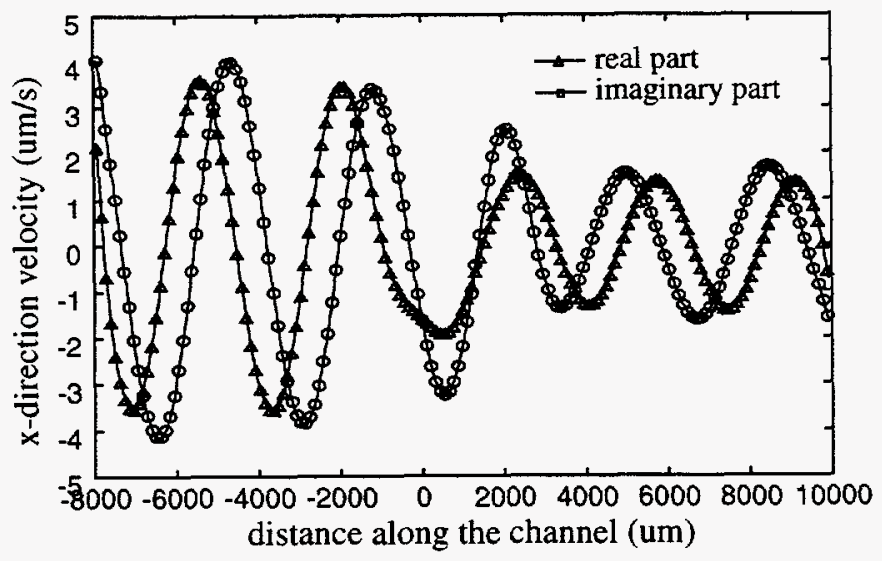

Figure 4: $x$-direction velocity along the center line of the channel

In Figure 5, the Reynolds stress resulting from the Figure 4 wave solution is shown. Notice that even though the integral of the stress along the length of the pump is positive, at some locations this stress is negative and then at other locations positive again. Therefore, even though the integral of the body force tends to drive fluid through the channel, internally this body force tends to also pull the fluid apart. In this paper, this effect will be referred to as fluid separation. If fluid separation occurs, the pump will be non-functional. This separation can only be balanced by an internal or external force which holds the fluid together. For example, if the fluid were under a static pressure or if viscous forces were very large, then separation could be avoided. 


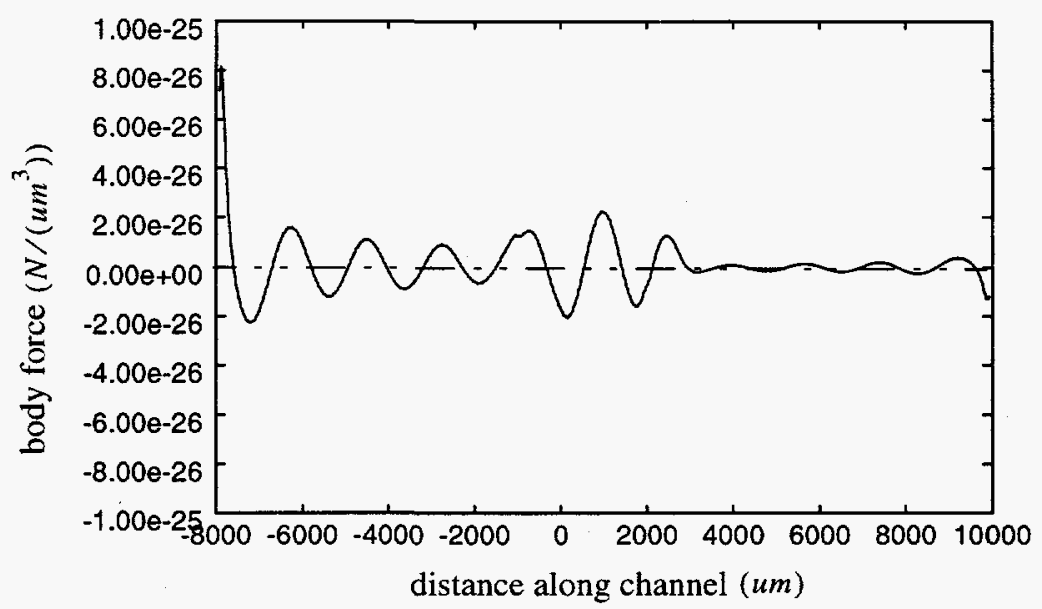

Figure 5: Body force in the $\mathrm{x}$ direction as a function of distance along the center line

By simulation, it was found that a left source relative phasing of $3 \pi / 2$ and amplitude of 1.0 will produce a maximum pressure drop across the channel. By holding the relative phasing of the second source to $3 \pi / 2$, by holding the amplitude of the secondary source to 1.0 and by varying $l_{w}$, an analysis of the optimal spacing of sources can be performed. Figure 6 shows the maximum pressure drop across the pump versus $l_{w} / \lambda$ where $\lambda=2 \pi / k_{l}$. For $l_{w} / \lambda=\frac{1}{8}, \frac{3}{8}, \frac{5}{8}, \ldots$ maximums occur. This corresponds to a spacing between the sources of $\frac{1}{4} \lambda, \frac{3}{4} \lambda, \frac{5}{4} \lambda, \ldots$.

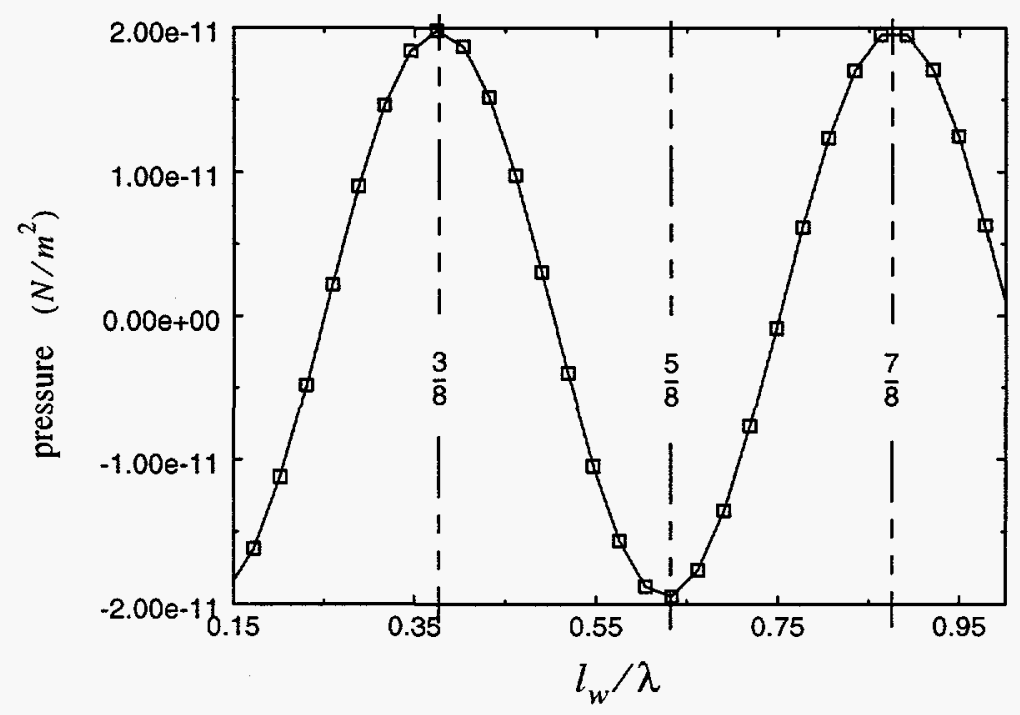

Figure 6: Pressure drop along the length of channel versus location of the sources. 


\section{CONCLUSIONS}

The analysis outlined in this paper makes a number of assumptions. First it was assumed that only first and second order effects are of importance. If the sources are driven too hard, higher order effects leading to turbulence and severe mixing will occur, and these assumptions will then be poor. It was also assumed that wall effects were small. Since first order acoustics were almost completely one dimensional, this was not an extremely poor assumption. Moreover, this assumption was a conservative one considering that its inclusion would help not hinder pumping.

Another assumption was that flow separation does not occur. The net body force in the pump will attempt to move the fluid left or right; however, internal to the fluid this same force will also attempt to produce fluid separation. If the fluid separates, no pumping action will occur. Instead, part of the fluid will be blown out of one end of the pump, part of the fluid will be blown out of the other end, and the rest will be trapped within. Separation could be limited if internal or external forces were sufficient to hold the fluid together. For example, if a net compressive force existed within the fluid which was greater than the repulsive body force, then pumping could occur.

Notice that the pressures in Figure 6 are very small. These pressures are for a right source velocity of $1 \mathrm{um} / \mathrm{s}$. If the sources are driven hard, this velocity will be much larger, and the Figure 6 pressure drop will be enhanced. Nevertheless, the exact level of excitation required to produce a significant pressure drop is as of yet unknown.

The concept of acoustic pumping is a novel one which should be explored further. The impact of developing this concept is substantial. Micro-pumps can be used in a number of applications ranging from micro-level cooling to micro-manipulation. However, the concept of acoustic pumping is still very theoretical. Experimental analysis is required.

\section{REFERENCES}

1. R.M. Moroney, R.M.White, and T.T. Howe, Fluid Motion Produced by Ultrasonic Lamb Waves, in Ultrasonics Symposium Proceedings, vol. 1, pp. 355-358, 1990.

2. S. Shiokawa and Y. Matsui, The Dynamics of SAW Streaming and it Application to Fluid Devices, in Material Research Society Symposium Proceedings, vol. 360, pp. 53$64,1995$.

3. W.L. Nyborg, Physical Acoustics: Principles and Methods, vol. 2, part B, Properties of Polymers and Nonlinear Acoustics, edited by W.P. Mason, Chapter 11, "Acoustic Streaming", pp. 265-330, 1965.

4. J. Lighthill, Acoustic Streaming, in Journal of Sound and Vibration, vol. 61, no. 3, pp. $391-418,1978$.

5. S. Temkin, Elements of Acoustics, Wiley, New York, 1981.

6. C.A. Brebbia, J.C.F. Telles, and L.C. Wrobel, Boundary Element Techniques, Theory and Applications in Engineering, Springer-Verlag, New York, 1987. 


\section{Distribution List}

\begin{tabular}{|c|c|c|c|c|}
\hline MS1413 & 1114 T.A.Michalske & $\begin{array}{l}\text { MS0321 } \\
\text { MS9202 }\end{array}$ & $\begin{array}{l}9200 \\
9202\end{array}$ & $\begin{array}{l}\text { W. Camp } \\
\text { R.J.Pryor }\end{array}$ \\
\hline MS 1425 & 1307 M.W.Scott & MS1111 & 9221 & S.S.Dosanjh \\
\hline MS0603 & 1313 C.P.Tigges & MS1111 & 9221 & C.C.Ober \\
\hline MS0603 & 1314 R.J.Shul & MS1110 & 9222 & R.C.Allen \\
\hline MS0603 & 1314 C.L.Willison & MS1110 & 9222 & D.E.Womble \\
\hline MS1425 & 1315 S.J.Martin & MS1110 & 9223 & D.Greenberg \\
\hline MS 1425 & 1315 G.C.Frye & MS1109 & 9224 & A.L.Hale \\
\hline MS1425 & 1315 M.A.Butler & MS1111 & 9225 & G.Heffelfinger \\
\hline MS1425 & 1315 W.K.Schubert & MS0441 & 9226 & R.Leland \\
\hline MS1080 & 1325 P.J.McWhorter & MS0819 & 9231 & J.M.McGlaun \\
\hline MS1080 & 1325 J.J.Allen & MS0820 & 9232 & P.Yarrington \\
\hline MS1080 & 1325 J.J.Sniegowski & & & \\
\hline & & MS0439 & 92341 & D.R.Martinez \\
\hline MS0303 & 2411 M.J.Forrestal & MS0439 & 9234 & J.L.Dohner (10) \\
\hline & & MS0439 & 9234 & D.J.Segalman \\
\hline MS0705 & 6114 D.F.Aldridge & MS0439 & 9234 & T.Hinnerichs \\
\hline MS1139 & 6421 A.W.Reed & MS0439 & 9234 & D.W.Lobitz \\
\hline & & MS0439 & 9234 & G.M.Reese \\
\hline MS1094 & 7524 M.C.Oborny & MS0557 & 9741 & T.J. \\
\hline MS0841 & 9100 P.J.Hommert & MS0557 & 9741 & P.S.Barney \\
\hline MS0833 & 9103 J.H.Biffle & MS0557 & 9741 & T.L.Paez \\
\hline MS0828 & 9104 R.Thomas & & & \\
\hline MS0826 & 9111 W.Hermina & MS9018 & $8940-2$ & 2 Central Tech. Files \\
\hline MS0834 & 9112 A.C.Ratzel & MS0899 & 4916 & Tech. Library (5) \\
\hline MS0835 & 9113 T.C.Bickel & MS0619 & 12690 & Review \& Aproval \\
\hline MS0835 & 9113 S.P. Burns & Desk (2) & & \\
\hline MS0827 & 9114 R.Griffith & & & For DOE/OST I \\
\hline MS0827 & 9114 C.C.Wong & MS0161 & $11500 \mathrm{~F}$ & Patent \& Licensing \\
\hline MS0827 & 9114 M.L. Hudson & Office & & \\
\hline MS0825 & 9115 W.H.Rutledge & & & \\
\hline MS0836 & 9116 C.W.Peterson & & & \\
\hline MS0443 & 9117/9118 H.S.Morgan & & & \\
\hline MS0437 & 9117/9118 C.Adams & & & \\
\hline MS0443 & 9117/9118 S.N.Burchett & & & \\
\hline MS0443 & 9117/9118 A.F.Fossum & & & \\
\hline $\mathrm{MSO4}$ & Key & & & \\
\hline
\end{tabular}

